\title{
Mapeamento das Políticas de Participação no Capital Social de Instituições Científicas e Tecnológicas (ICT) em Empresas
}

\author{
Mapping of the Policy of Participation in Social Capital of Scientific and \\ Technological Institutions (ICT) in Companies
}

\author{
Leonardo Miranda Frossard ${ }^{1}$ \\ Rita Pinheiro-Machado² \\ Flávia Lima do Carmo ${ }^{3}$ \\ ${ }_{1}^{1}$ Universidade Federal de Juiz de Fora - UFJF/PROFNIT - Ponto Focal UFRJ, Rio de Janeiro, RJ, Brasil \\ ${ }^{2}$ Instituto Nacional da Propriedade Industrial - INPI/PROFNIT - Ponto Focal UFRJ, Rio de Janeiro, RJ, Brasil \\ ${ }^{3}$ Universidade Federal do Rio de Janeiro - UFRJ/PROFNIT - Ponto Focal UFRJ, Rio de Janeiro, RJ, Brasil
}

\begin{abstract}
Resumo
As universidades têm ampliado o seu escopo de atuação, desde sua criação no século XI, agregando à sua missão o ensino e a pesquisa; e recentemente, a sociedade tem demandado um papel mais atuante da universidade no desenvolvimento econômico e social, internalizando o conceito de "Universidade Empreendedora". A pesquisa teve o intuito de identificar e realizar um estudo comparativo das ICT públicas e privadas brasileiras que possuem cláusulas de participação acionária em empresas. Para atingir os objetivos do trabalho foi realizada uma análise nas políticas de inovação para identificar as ICT que possuem cláusulas de políticas de participação minoritária no capital social de empresas. Os resultados demonstram que, por ser um tema pouco explorado nas ICT brasileiras, apenas sete delas possuem cláusulas em suas políticas de inovação. O trabalho sugere que as entidades do Sistema Nacional de Inovação se articulem para oferecer capacitação e produzir material informativo sobre como as ICT podem implantar ações de investimento em empresas.
\end{abstract}

Palavras-chave: Instituições Científicas e Tecnológicas. Lei de Inovação. Participação Acionária.

\begin{abstract}
Universities have expanded their scope of action since their creation in $11^{\text {th }}$ century, adding their mission to teaching, research and recently, society has demanded a more active role of the university in economic and social development, internalizing the concept of "University Entrepreneur". The research aimed to identify and conduct a comparative study of Brazilian public and private ICTs that have clauses of shareholding participation in companies. In order to reach the objectives of the work, an analysis was made in the innovation policies to identify the ICTs that have clauses of policies of minority participation in the social capital of companies. The results show that, because it is a topic not explored in the Brazilian ICT, only seven have clauses in their innovation policies. The work suggests that the entities of the National Innovation System, articulate to offer training and produce informative material on how ICT can implement investment actions in companies.
\end{abstract}

Keywords: Scientific and Technological Institutions. Innovation Law. Ownership Participation.

Área Tecnológica: Empreendedorismo. Inovação. Investimento. 


\section{Introdução}

A sociedade moderna vem demandando uma nova atuação para as universidades. Desde sua criação no século XI, as universidades passaram por diversas mudanças. Inicialmente, o foco era a transmissão do conhecimento. No século XVII, veio a primeira revolução, com a introdução da pesquisa agregada ao ensino. Nos anos 1990, com a observação do modelo de universidades americanas, como MIT (Massachussetts Institute of Tecnology), Stanford e Havard, foi internalizado à sua missão o conceito de "Universidade Empreendedora", no qual a universidade passa a capitalizar o conhecimento (ETZKOWITZ, 1998).

Neste contexto, surge o conceito de Sistema Nacional de Inovação (SNI), que, na visão de Freeman (1995), é um conjunto de instituições, atores e mecanismos que interagem e contribuem para o desenvolvimento tecnológico de um país. Os principais atores do SNI brasileiro são as universidades, cujo papel é transmitir o conhecimento e a pesquisa; a empresa, que atua na transformação do conhecimento em produto; e o governo, que é responsável por criar e fomentar políticas públicas em Ciência, Tecnologia e Inovação (CT\&I).

Com o intuito de potencializar a atuação dos atores de SNI brasileiro, foi aprovada a Lei n. 13.243, de 11 de janeiro de 2016, e recentemente foi assinado o Decreto n. 9.283, de 7 de fevereiro de 2018, que regulamenta e altera as leis para estabelecer medidas de incentivo à inovação e à pesquisa científica e tecnológica no ambiente produtivo. No decreto, dentre as regulamentações está a Seção II, que discorre sobre a participação minoritária no capital social de empresas e dos fundos de investimento.

Como essa é uma situação nova para as Instituições Científicas e Tecnológicas (ICT) ${ }^{1}$ brasileiras, o presente trabalho tem o intuito de, inicialmente, identificar as ICT que possuem cláusulas de participação minoritária no capital social de empresas e realizar uma análise comparativa.

\section{Metodologia}

Neste trabalho, por meio de uma pesquisa qualitativa e quantitativa, inicialmente foi realizada uma revisão bibliográfica dos conceitos relacionados ao tema. Tais levantamentos permitem classificar como método de investigação a pesquisa bibliográfica que, na percepção de Gil (2007, p. 44) "é desenvolvida com base em material já elaborado, constituído principalmente de livros e artigos científicos".

Além da pesquisa bibliográfica foi realizada uma análise documental das pesquisas Highlightsof AUTM's U.S. Licensing Activity Survey FY2015 e do Formulário para Informações sobre a Política de Propriedade Intelectual das Instituições Científicas, Tecnológicas do Brasil (FORMICT) - ano base 2016. Segundo Gil (2007, p. 45), a pesquisa documental é um método de investigação que se vale de dados secundários e materiais que não receberam ainda um tratamento analítico ou que ainda podem ser reelaborados de acordo com os objetivos da pesquisa.

Para a identificação das ICT que possuem políticas de participação minoritária no capital social de empresas foi realizado um levantamento dentre as instituições participantes do FOR-

\footnotetext{
${ }^{1}$ Instituições Científicas e Tecnológicas (ICT) - órgão ou entidade da administração pública direta ou indireta ou pessoa jurídica de direito privado sem fins lucrativos legalmente constituída sob as leis brasileiras, com sede e foro no País, que inclua em sua missão institucional ou em seu objetivo social ou estatutário a pesquisa básica ou aplicada de caráter científico ou tecnológico ou o desenvolvimento de novos produtos, serviços ou processos (Brasil, 2016).
} 
MICT e que são associadas à Associação Nacional de Entidades Promotoras de Empreendimentos Inovadores (Anprotec) ${ }^{2}$, visto que o principal meio de apoio e formação de empresas nascentes de base tecnológica nas ICT são as incubadoras de empresas e parques tecnológicos.

Dentre as 278 instituições respondentes do FORMICT, 81 são associadas à Anprotec ou possuem incubadoras de empresas ou parques tecnológicos (ANPROTEC, 2018). Desta forma, foi realizado um levantamento no site dessas instituições (Vide Anexo A) com o intuito de localizar as Políticas de Inovação e Políticas de Propriedade Intelectual, e dentre as identificadas, as que possuem cláusulas de participação minoritária no capital social de empresas.

Posteriormente, foi realizada uma análise documental nas políticas de inovação ou de Propriedade Intelectual, com o objetivo de avaliar e fazer um estudo comparativo das cláusulas de participação minoritária no capital social das ICT selecionadas.

\section{Resultados e Discussão}

\subsection{O Sistema Nacional de Inovação}

O Sistema Nacional de Inovação Brasileiro é representado pelos seguintes atores: Instituições Científicas e Tecnológicas (ICT) - são organizações públicas ou privadas que atuam em atividades de pesquisa e podem transferir os conhecimentos gerados para as empresas e a sociedade; Empresas - são organizações que têm o papel de desenvolver, produzir e comercializar produtos e serviços. A empresa é a instituição responsável por implementar as inovações; Governo - a atribuição do governo no SNI é a regulamentação, o fomento por meio de políticas públicas $e$ a articulação entre os atores; Entidades - são organizações sem fins lucrativos que atuam na representação e articulação dos atores do SNI. Dentre as instituições, destacam-se: Fortec, Anprotec e Anpei, entre outras; Habitats e Suporte - são instituições que apoiam ações nas áreas de propriedade intelectual, transferência de Tecnologia, apoio a empreendedores, startups, empresas e interação entre as universidades, o governo e as empresas. Dentre as instituições, se destacam os Núcleos de Inovação Tecnológica (NIT), as Incubadoras e Aceleradoras de Empresas, os Parques Tecnológicos, o Sebrae, entre outras; e os Investidores - prospectam e analisam novos projetos, modelos de negócios e empresas com potencial de crescimento (ANPEI, 2018).

O conceito de SNI surgiu dos modelos de interação universidade-empresa-governo, como o Triângulo de Sábato, e posteriormente, com a Tríplice Hélice. O Triângulo de Sábato foi apresentado em 1968, por Jorge Sábato e Natalio Botana, no trabalho "Ciência e Tecnologia no Desenvolvimento Futuro da América Latina", na World Order Models Conference, realizada em Bellagio, na Itália (PLONSKI, 1995). A recomendação era que, para a América Latina superar o subdesenvolvimento deveria implementar ações decisivas no campo da ciência e tecnologia. O modelo foi apresentado graficamente como um triângulo, no qual o governo ocupa a parte superior e a universidade e a empresa os vértices da base. No modelo estão previstos três tipos de interações: intrarrelações - são relações que ocorrem entre o governo, a universidade e a empresa; inter-relações - que acontecem deliberadamente entre as partes; e extrarrelações - que

\footnotetext{
${ }^{2}$ Anprotec - Associação Nacional de Entidades Promotoras de Empreendimentos inovadores: a associação reúne incubadoras de empresas, parques tecnológicos, aceleradoras, coworkings, instituições de ensino e pesquisa, órgãos públicos e outras instituições ligadas ao empreendedorismo e à inovação. A instituição atua na articulação entre seus associados, com promoção de capacitações, articulações de políticas públicas e geração e disseminação do conhecimento.
} 
ocorrem entre a sociedade e o exterior, por meio de intercâmbio científico, comércio externo $e$ adaptação de tecnologias.

A tríplice hélice é uma evolução do modelo de interação entre os atores, visto que, além das conexões, estes passam a desempenhar funções dos outros dois, e considera a constituição de redes interativas entre as instituições que configuram as hélices do modelo (STAL; FUJINO, 2005). A tríplice hélice surgiu da observação do MIT e foi apresentada por Henry Etzkowitz nos anos 90, criando o conceito de universidade empreendedora (ETZKOWITZ, 1998).

Partindo do pressuposto que as universidades empreendedoras são indutoras do desenvolvimento econômico e social (MARGINSON; CONSIDINE, 2004; ETZKOWITZ, 2004; ETZKOWITZ; KLOFSTEN, 2005), há duas perspectivas: a primeira apresenta a ideia da Universidade-Empresa com atuação nas dimensões econômicas, acadêmicas e institucionais; e a segunda, trata da universidade empreendedora, que se diferencia das demais, por três características: a) disseminação do empreendedorismo inovador na comunidade acadêmica; b) interação entre universidade-empresa-governo por meio de escritórios de transferência de tecnologia; e c) expressivo número de pesquisadores, alunos e indivíduos com conhecimento para criação de empresas nascentes de base tecnológica.

A visão da Tríplice Hélice apresentada por Etzkowitz e Leydesdorff (2000), na qual a Universidade assume o papel de empreendedora, formando mão de obra especializada, atuando na pesquisa e modificando suas estruturas para levar a pesquisa desenvolvida para o mercado, por meio da criação e desenvolvimento de empresas nascentes de base tecnológica, de fato ainda apresenta gargalos que impedem o devido transbordamento da pesquisa para o mercado de forma efetiva.

\subsection{Análise dos Sistemas Nacionais de Inovação Norte-americano e Brasileiro}

Nos anos 80, o sistema de inovação norte-americano sofreu importantes alterações, devido à perda de competitividade da economia frente a ascensão do Japão. Assim, a transformação veio em duas vertentes: a primeira com a criação de instituições como a Technology Administration (vinculada ao Departamento de Comércio), agência que atuava no estímulo à comercialização das pesquisas científicas por meio de Transferência de Tecnologia e monitoramento de três outras agências: National Institute of Standard Technology - NIST, National Technical Information Service - NTIS e Office of Technology Policy - OPT. A segunda vertente veio com o marco regulatório para estímulo a investimentos e fomento à inovação, com a edição e criação de leis como: Stevenson-Wydler Technology Innovation Act of 1980; Bayh-Dole Act of 1980; Small Business Innovation Development Act of 1982³; Federal Technology Transfer Act of 1986; National Cooperative Research Act of 1984, ampliada pela National Cooperative Research and Production Act of 1993 e Omnibus Trade and Competitiveness Act of 1988. As medidas jurídico-institucionais tiveram impacto para que os resultados de pesquisa das universidades americanas, laboratórios federais e outras instituições gerassem produtos comercializáveis para a aplicação na indústria (MATTOS; ABDAL, 2010).

\footnotetext{
${ }^{3}$ Denominado Programa SBIR que estimulou a atração da Indústria de Venture Capital para apoio as empresas nascentes (MATOS; ABDAL, 2010).
} 
As iniciativas oportunizaram a disseminação de conhecimento e informações, o fomento à transferência de tecnologia com potencial de mercado e à criação de parcerias para desenvolvimento de mecanismos de Seed Capital e Venture Capital. Em 1982, foi criada a Lei de Desenvolvimento e Inovação para Pequenas Empresas (Small Business Innovation Development Act of 1982 - SBIR). O Programa SBIR estimulou a atração da Indústria de Venture Capital para apoio a empresas nascentes (MATTOS; ABDAL, 2010).

As medidas adotadas trouxeram resultados nos indicadores de C,T\&I norte-americanos. Os dispêndios em Pesquisa e Desenvolvimento (P\&D), no ano de 2014, foram de US\$479,40 bilhões, com dispêndios em relação ao Produto Interno Bruto (PIB) de 2,76\% (MCTIC, 2017). Com relação à fonte desses recursos, a Association of University Technology Managers (AUTM), instituição americana para apoio a escritórios de transferência de tecnologia, traz na publicação Highlightsof AUTM's U.S. Licensing Activity Survey FY2015 informações sobre as universidades norte-americanas e informa que, dentre as universidades respondentes da pesquisa, foram identificados investimentos em P\&D de US\$ 66,5 bilhões, e destes US\$ 39,2 bi vieram de financiamento federal $(58,9 \%)$, US $\$ 4,9$ bi de financiamento da indústria $(7,4 \%)$ e US $\$ 22,4$ bi de outras fontes $(33,7 \%)$.

De acordo com a mesma Associação, foram depositadas pelas universidades norte-americanas 24.723 patentes e foram concedidas 6.680. Os licenciamentos de tecnologia, no mesmo ano, atingiram 6.395 licenças, com 1.547 opções executadas e 654 licenças executadas com participação acionária. No mesmo período, também surgiram 1.012 startups, 879 novos produtos criados e vendas líquidas de produtos de US\$28,7 bilhões (AUTM, 2015).

No Brasil, com o intuito de estimular um ambiente cooperativo para a produção científica, tecnológica e de inovação, foi promulgada a Lei n. 10.973/2004. A Legislação foi inspirada na Lei de Inovação Francesa ${ }^{4}$ e no Bayh-Dole Act norte-americano. Mais recentemente, em janeiro de 2016 foi sancionada a Lei n. 13.243 e regulamentado, em fevereiro de 2018, o novo marco legal de Ciência, Tecnologia e Inovação com atualizações para o fortalecimento da pesquisa e da produção do conhecimento, buscando corrigir falhas para o estímulo à interação Universidade-Empresa (RAUEN, 2016).

De acordo com dados do Ministério da Ciência, Tecnologia, Inovação e Comunicação (MCTIC, 2018), os dispêndios brasileiros em P\&D foram de US $\$ 42,60$ bilhões e os dispêndios em relação ao PIB foram de 1,27\%, ambos em 2014. Com o objetivo de obter informações acerca das ações das ICT brasileiras no campo da propriedade intelectual e transferência de tecnologia, a Lei n. 13.243, determina em seu Art. 17 que:

A ICT pública deverá, na forma de regul'amento, prestar informações ao Ministério da Ciência, Tecnologia e Inovação.

Parágrafo Único - Aplica-se o disposto no caput à ICT privada beneficiada pelo poder público, na forma desta Lei.

Atendendo ao Art. 17, o MCTIC aplica, anualmente, o Formulário para Informações sobre a Política de Propriedade Intelectual das Instituições Científicas, Tecnológicas do Brasil (FORMICT). O FORMICT tem como objetivo a coleta de informações relativas às políticas de propriedade intelectual das instituições científicas e tecnológicas (ICT) do Brasil.

${ }^{4}$ Loi n. 82-610 du 15 juillet 1982 d'orientation et de programmation pour la recherche et le développement technologique de la France. 
Em 2016, dentre as 278 instituições respondentes, 193 eram públicas e 85 privadas. Das 278 ICT, 208 afirmaram que o Núcleo de Inovação Tecnológica (NIT) estava implementado, 46 em implantação e 24 não implementado. De acordo com o Formict ano-base 2016, foram depositados 2.390 pedidos de patentes e 813 foram concedidas. Dentre todas as instituições, apenas 42 públicas e 16 privadas executaram contratos de tecnologia, representando, respectivamente, $21,76 \%$ e $18,82 \%$. Os contratos negociados correspondem a $\mathrm{R} \$ 437,90$ milhões, no ano de 2016 (MCTIC, 2017).

Desta forma, realizando uma análise comparativa das pesquisas Highlightsof AUTM's U.S. Licensing Activity Survey FY2015 e Formict 2016 constata-se que, enquanto na pesquisa nas universidades norte-americanas são coletados dados sobre os investimentos em $\mathrm{P} \& \mathrm{D}$, número de patentes depositadas e concedidas, número de licenciamentos (com opções executadas e com participação acionária) e número de startups criadas (com número de produtos criados e volume de vendas em dólares), o Formict trata do estágio de implantação dos NIT (implantados e em implantação); ICT com pedidos de proteção intelectual e análise dos pedidos de proteção; ICT com contratos de transferência de tecnologia, análise dos contratos de transferência de tecnologia e rendimentos obtidos com os contratos.

\subsection{O Novo Marco Legal e seus Desdobramentos}

A Lei n. 13.243, de 11 de janeiro de 2016, que dispõe sobre os estímulos ao desenvolvimento científico, à pesquisa, à capacitação científica e tecnológica e à inovação e altera uma série de outras leis, com o objetivo de potencializar a atuação dos atores do Sistema Nacional de Inovação, traz em seu texto benefícios para as ICT atuarem de forma mais autônoma e com maior agilidade.

Para tanto, após diversas discussões nas Comissões de Ciência, Tecnologia, Inovação, Comunicação e Informática (CCT), Comissões de Constituição, Justiça e Cidadania (CCJ) e de Assuntos Econômicos (CAE), foi assinado o Decreto n. 9.283, de 7 de fevereiro de 2018, que regulamenta a Lei n. 10.973, de 2 de dezembro de 2004, a Lei n. 13.243, de 11 de janeiro de 2016, o art. 24, $\S 3^{\circ}$, e o art. $32, \S 7^{\circ}$, da Lei n. 8.666, de 21 de junho de 1993 , o art. $1^{\circ}$ da Lei n. 8.010, de 29 de março de 1990, e o art. $2^{\circ}$, caput, inciso I, alínea "g", da Lei n. 8.032, de 12 de abril de 1990, e altera o Decreto n. 6.759, de 5 de fevereiro de 2009, para estabelecer medidas de incentivo à inovação e à pesquisa científica e tecnológica no ambiente produtivo, com vistas à capacitação tecnológica, ao alcance da autonomia tecnológica e ao desenvolvimento do sistema produtivo nacional e regional (BRASIL, 2016).

No Decreto n. 9.283/2018, dentre as regulamentações está a Seção II, que discorre sobre a participação minoritária no capital e dos fundos de investimento. O Art. $4^{\circ}$ estabelece que:

Ficam as ICT públicas integrantes da administração pública indireta, as agências de fomento, as empresas públicas e as sociedades de economia mista autorizadas a participar minoritariamente do capital social de empresas, com o propósito de desenvolver produtos ou processos inovadores que estejam de acordo com as diretrizes e as prioridades definidas nas políticas de ciência, tecnologia, inovação e de desenvolvimento industrial.

$\S 1^{\circ} \mathrm{A}$ entidade de que trata o caput estabelecerá a sua política de investimento direto $e$ indireto, da qual constarão os critérios e as instâncias de decisão e de governança, e que 
conterá, no mínimo: I - a definição dos critérios e dos processos para o investimento $e$ para a seleção das empresas; II - os limites orçamentários da carteira de investimentos; III - os limites de exposição ao risco para investimento; IV - a premissa de seleção dos investimentos e das empresas-alvo com base: a) na estratégia de negócio; b) no desenvolvimento de competências tecnológicas e de novos mercados; e c) na ampliação da capacidade de inovação; $\mathrm{V}$ - a previsão de prazos e de critérios para o desinvestimento; VI - o modelo de controle, de governança e de administração do investimento; e VII - a definição de equipe própria responsável tecnicamente pelas atividades relacionadas com a participação no capital social de empresas.

A regulamentação trará para as ICT uma segurança jurídica para atuar de forma mais efetiva na transferência de tecnologia e apoio a startups, por meio das ações de empreendedorismo, incubadoras de empresas, parques tecnológicos e na participação minoritária do capital social de empresas de base tecnológica.

\subsection{Modalidades de Investimentos em Empresas de Base Tecnológica}

As Empresas de Base Tecnológica são organizações que aplicam conhecimentos científicos e tecnológicos e técnicas pioneiras ou avançadas para criação e desenvolvimento de novos produtos e processos (FINEP, 2018). Para Meirelles, Pimenta e Nascimento Rebelatto (2008), uma das alternativas de financiamento para as empresas nascentes de base tecnológica está no aporte de recursos oriundos de fundos de Private Equity ou Venture Capital.

\subsection{A Indústria de Private Equity e Venture Capital}

A indústria de Private Equity e Venture Capital (PE/VC) surgiu nos EUA para financiar as inovações tecnológicas advindas da $2^{a}$ Guerra Mundial e impulsionou a geração de riqueza norte-americana desde a década de 50. As primeiras iniciativas de investimentos vieram com a criação da American Research Development (ARD) e pela corrida espacial, com o lançamento da American Research Project Agency (ARPA), que posteriormente se tornou DARPA - incluindo Defesa. A criação destas agências foi a base para o surgimento de disciplinas de Ciência da Computação em importantes universidades norte-americanas (Utah, Instituto Carnegie-Mellon, Califórnia e Stanford). As iniciativas foram o embrião para o surgimento do movimento de garage companies ${ }^{5}$ e do Vale do Silício ${ }^{6}$ (ABDI, 2011).

A indústria de PE/VC, como apresentado na Figura 1, apresenta estágios e modalidades de investimentos, a saber: a) Seed - as empresas estão na fase de concepção e os recursos são para a criação e desenvolvimento do produto ou prova de conceito; b) Startup - fase de desenvolvimento e validação do produto e do modelo de negócios da empresa, e os recursos são para investimento em marketing; c) Venture Capital - dividido entre Early Stage - no qual o produto já está desenvolvido, modelo de negócios validado e os investimentos são para iniciar as vendas; e Large Stage - necessidade de investimento para expansão rápida das vendas do produto; d) Private Equity - dividido em dois estágios: Growth - empresas maduras que necessitam de investimentos para entrada em novos mercados ou aquisição de outras empresas; e

\footnotetext{
${ }^{5}$ Garage Companies - empresas criadas no fundo das garagens das casas por estudantes norte-americanos. As mais conhecidas foram Hewlett-Packard, Apple, Microsoft (ABDI, 2011).

${ }^{6}$ Vale do Silício - é uma região que abrange várias cidades (São Francisco, Palo Alto, Santa Clara) no estado da Califórnia, nos EUA. No Vale do Silício estão instaladas muitas empresas de tecnologia, como Apple, Google, Facebook, HP, Symantec, Intel, dentre outras (ABDI, 2011).
} 
Later Stage - empresas em estágio avançado de desenvolvimento e os investimentos são para a reestruturação do negócio; e) Empresas de capital aberto, com ações negociadas na bolsa de valores, que utilizam a modalidade de investimentos Mezanino - empresas com alta geração de caixa, por meio de dívidas subordinadas e que utilizam instrumentos híbridos de financiamento (debêntures conversíveis e direitos de subscrição). São instrumentos de dívidas, podendo ser total ou parcialmente conversíveis em ações e Private Investiment in Public Equity (PIPE) - operação de aquisição de um volume considerável do capital de empresa (mínimo 20\%) já listada na bolsa (ABDI, 2011).

Figura 1 - Estágios e Modalidades de Investimentos de PE/VC

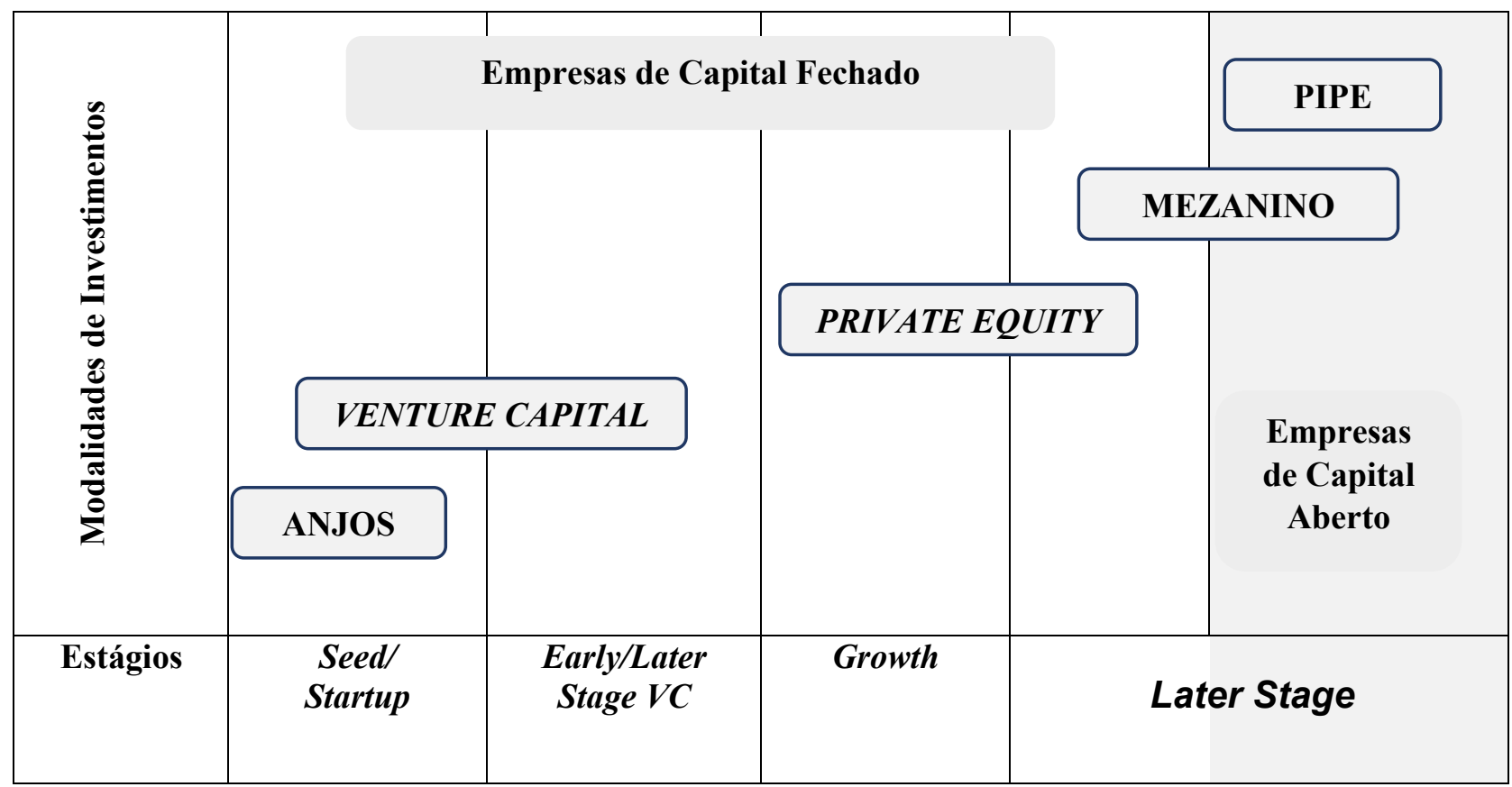

Fonte: ABDI (2011)

A indústria de PE/VC possui quatro atores principais: os investidores, as organizações gestoras, as instituições de investimentos (fundos de PE/VC) e as empresas investidas.

Como apresentado na Figura 2, o ciclo de investimento da indústria de PE/VC inicia-se com $\mathrm{o}$ aporte de recursos nos fundos de PE/VC. As empresas e startups buscam recursos e apresentam seus modelos de negócios para as Companhias de PE/VC. Estas instituições realizam uma avaliação criteriosa das empresas que possuem potencial de receber investimentos. As selecionadas recebem o aporte de capital (investimento) dos Fundos de PE/VC e são acompanhadas pelas Companhias de PE/VE. Como o foco dos Fundos de PE/VC é o de obter retorno financeiro sobre os investimentos realizados, após determinado período de tempo, os fundos realizam a saída (que pode ser por meio de Initial Public Offering (IPO), abertura de capital na bolsa de valores, Fusão, Aquisição, Liquidação) (MEIRELLES; PIMENTA; NASCIMENTO REBELATTO, 2008). 
Figura 2 - Principais atores da Indústria de PE/VC

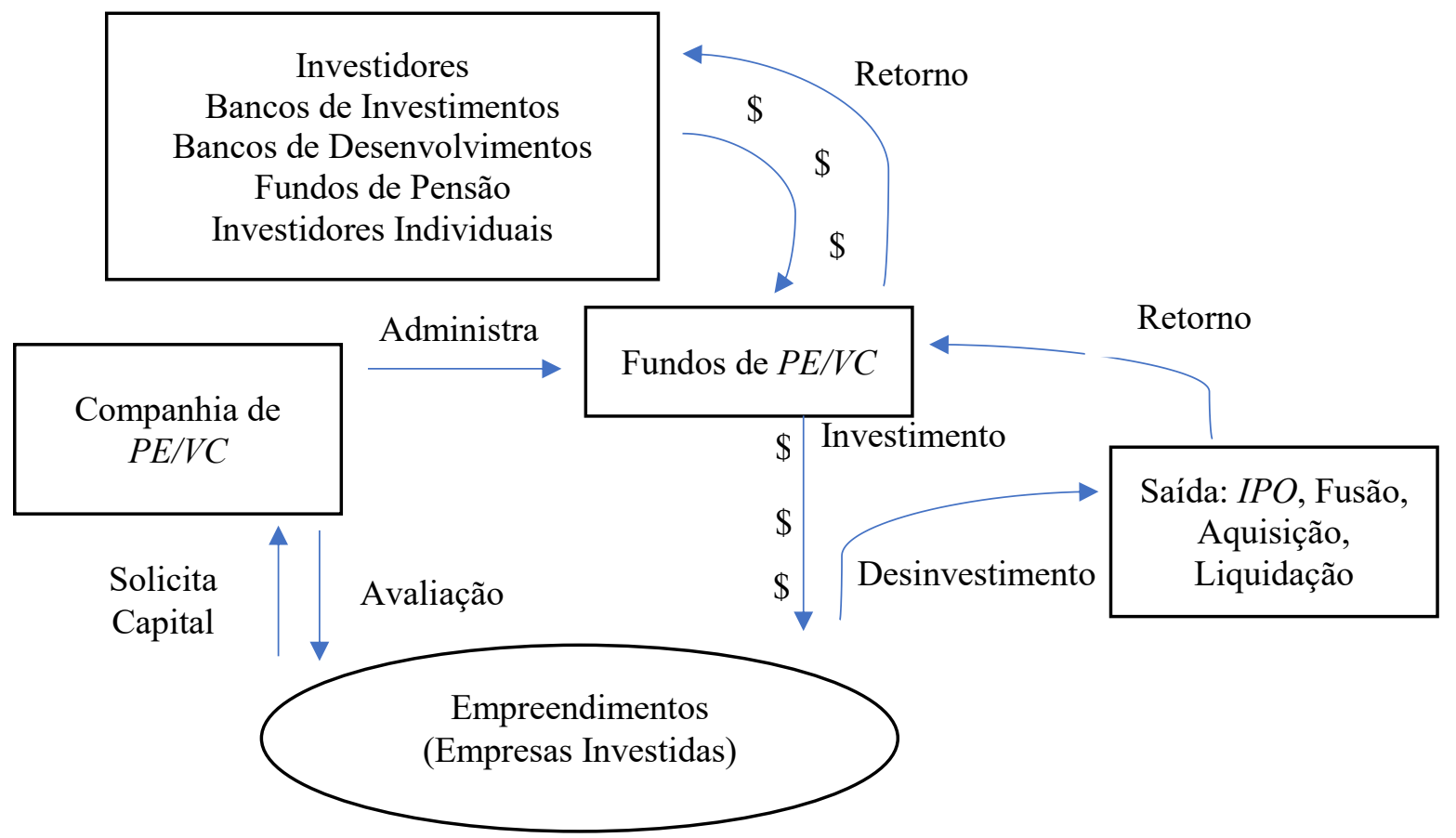

Fonte: Meirelles, Pimenta e Nascimento Rebelatto (2008)

Ainda, de acordo com Meirelles, Pimenta e Nascimento Rebelatto (2008), o aporte de recursos de fundos de $P E / V C$ é interessante, pois profissionaliza o negócio. Os gestores trazem uma série de benefícios, como: rede de relacionamento, abertura de oportunidades de crédito, visão de outras experiências de investimento do fundo, dentre outras.

Por se tratar de empresas em estágio inicial de desenvolvimento, as empresas de base tecnológica apoiadas pelas ICT brasileiras estão no estágio de Seed/Startup ou Early/Later Stage, podendo receber investimentos anjos ${ }^{7}$ e Venture Capital. Os Fundos de Investimentos em Participação (FIP) em capital semente são o instrumento mais utilizado pela indústria de PE/VC para aporte de investimentos.

\subsection{Fundos de Investimentos em Participações - FIP}

Os Fundos de Investimentos em Participações - FIP são produtos ofertados pela BM\&FBovespa - Bolsa de Valores. O FIP é um conjunto de recursos financeiros captados junto a investidores e que são destinados à aplicação em empresas abertas, fechadas ou sociedades limitadas. Estes fundos são constituídos sob a forma de condomínios fechados e as cotas só podem ser resgatadas ao término do fundo ou quando há liquidação. Os Fundos de Investimentos em Participação buscam gerar valor para as empresas investidas e implementar práticas de governança corporativa (BM\&F-Bovespa, 2018).

Os FIPs podem ser classificados como: Capital Semente, Empresas emergentes, Infraestrutura e Produção Econômica intensiva em Pesquisa, Desenvolvimento e Inovação e Multiestratégia, como apresentado no Quadro 1.

\footnotetext{
${ }^{7}$ Investimento Anjo - São investimentos aportados, geralmente, por um grupo de investidores com significativo volume de capital (pessoas físicas). Os investidores anjos possuem larga experiência executiva ou já passaram por rodadas de investimento e desinvestimento de instituição de PE/VC (ABDI, 2011).
} 
Quadro 1 - Classificação dos Fundos de Investimentos em participação

\begin{tabular}{lll}
\hline \multicolumn{1}{c}{ Tipo } & \multicolumn{1}{c}{ Se tor } & \multicolumn{1}{c}{ Receita Bruta } \\
\hline FIP - Capital Semente & Companhias ou Sociedade Limitada & Até R\$16 milhões \\
FIP - Empresas Emergentes & Companhias ou Sociedade Limitada & Até R\$16 milhões \\
& $\begin{array}{l}\text { Títulos de emissão de sociedades anônimas, de capital } \\
\text { aberto ou fechado, que desenvolvam, respectivamente, }\end{array}$ & Cada FIP-IE e FIP-P,D\&I deve ter, no mínimo, \\
& novos projetos de infraestrutura ou de produção & cinco cotistas, sendo que cada cotista não pode \\
FIP - Infraestrutura (FIP-IE) e FIP & econômica intensiva em pesquisa, desenvolvimento e & deter mais de 40\% das cotas emitidas pelo FIP- \\
Produção Econômica intensiva em Pesquisa, & inovação nas áreas de energia, transporte, água e & IE ou pelo FIP-P,D\&I ou auferir rendimento \\
Desenvolvimento e Inovação (FIP-P,D\&I) & $\begin{array}{l}\text { saneamento básico, irrigação e outras áreas prioritárias superior a 40\% do rendimento do fundo. } \\
\text { para o Poder Executivo Federal. }\end{array}$ & \\
& $\begin{array}{l}\text { Não se classificam nas demais categorias por admitir o Até 100\% de seu capital subscrito em ativos no } \\
\text { investimento em diferentes tipos e portes de }\end{array}$ & $\begin{array}{l}\text { exterior, porém, neste caso, são destinados } \\
\text { exclusivamente a investidores profissionais. }\end{array}$ \\
\hline FIP - Multiestratégia & sociedades investidas. &
\end{tabular}

Fonte: Desenvolvido pelos autores deste artigo, com dados da BM\&F-Bovespa

Como previsto no artigo $4^{\circ}$ do Decreto n. 9.283, de 7 de fevereiro de 2018, as ICT estão

[...] autorizadas a participar minoritariamente do capital social de empresas, com o propósito de desenvolver produtos ou processos inovadores que estejam de acordo com as diretrizes e as prioridades definidas nas políticas de ciência, tecnologia, inovação e de desenvolvimento industrial.

Desta forma, o tipo de Fundo de Investimento em Participação mais adequado para as ICT é o FIP - Capital Semente.

\section{Resultados}

Com o objetivo de identificar as ICT que possuem cláusulas de participação no capital social de empresas, foi realizado um levantamento entre as 278 ICT respondentes do Formict no ano de 2016 e as associadas à Anprotec, visto que o principal meio de criação e apoio às empresas nascentes de base tecnológica são as incubadoras de empresas e parques tecnológicos. Como resultado, foi identificado que 81 ICT são associadas à Anprotec.

Posteriormente, foi realizada uma análise no site das instituições, com o intuito de localizar suas políticas de inovação ou de propriedade intelectual. Dentre as 81 , foram localizadas as resoluções em 43 instituições, e destas, 14 possuem Políticas de Inovação e 29 possuem Política de Propriedade Intelectual.

Por fim, após a análise das Políticas de Inovação e de Proporiedade Intelectual das ICT selecionadas, foram identificadas sete ICT que possuem cláusulas de participação minoritária no capital social de empresas, a saber: Universidade Federal do Ceará - UFC, Centro Federal de Educação Tecnológica de Minas Gerais - CEFET-MG, Universidade Federal de São João Del Rei - UFSJ, Universidade Federal de Uberlândia - UFU, Universidade Federal de Viçosa - UFV, Instituto Federal do Mato Grosso do Sul - IFMS e Instituto Nacional de Metrologia, Qualidade e Tecnologia - INMETRO. 
Quadro 2 - ICTs que possuem cláusulas de participação minoritária no Capital Social de Empresas

\begin{tabular}{|c|c|c|c|c|c|}
\hline $\mathrm{N}^{\mathbf{0}}$ & ICT & Sigla & UF Ano & Resolução & Cláus ula de Participação no Capital Social \\
\hline 1 & $\begin{array}{c}\text { Universidade Federal do } \\
\text { Ceará }\end{array}$ & UFC & CE 2017 & $\begin{array}{l}\text { Resolução no } \\
\text { 38/CONSUNI, de } 18 \text { de } \\
\text { Agosto de } 2017\end{array}$ & $\begin{array}{l}\text { CAPÍTULO - VI DO LICENCIAMENTO E TRANSFERÊNCIA DE TECNOLOGIA Art. } \\
\text { 14. É facultado à UFC celebrar contratos de transferência de tecnologia e de licenciamento } \\
\text { para outorga de direito de uso ou de exploração de criação em que seja titular ou cotitular por } \\
\text { ela desenvolvida isoladamente ou por meio de parceria, a título exclusivo e não exclusivo.... } \S \\
5^{\circ} \text { A UFC, a seu exclusivo critério, poderá negociar como forma de remuneração pelo } \\
\text { licenciamento ou transferência de criação de sua titularidade, participar minoritariamente do } \\
\text { capital social de empresa ou usufruto de ações ou quotas da empresa licenciada, na forma } \\
\text { estabelecida no art. } 5^{\circ}, \S 1^{\circ} \text { a } 6^{\circ} \text { da Lei } n^{\circ} 13.246 / 16\end{array}$ \\
\hline 2 & $\begin{array}{l}\text { Centro Federal de } \\
\text { Educação Tecnológica } \\
\text { de MG }\end{array}$ & CEFET-MG & J MG 2018 & $\begin{array}{c}\text { Resolução } n^{\circ} \text { CD-027/18, } \\
\text { de } 07 \text { de Maio de } 2018\end{array}$ & $\begin{array}{l}\text { CAPÍTULO X - DA PARTICIPAÇÃO NO CAPITAL SOCIAL DE EMPRESAS Art. } 32 \text { - } \\
\text { O CEFET-MG poderá, nos termos da legislação vigente, participar minoritariamente do capital } \\
\text { social de empresas, por meio de contribuição financeira e não financeira, desde que } \\
\text { economicamente mensurável, com o propósito de desenvolver produtos, processos ou serviços } \\
\text { inovadores que estejam de acordo com as diretrizes e prioridades definidas na Política de } \\
\text { Ciência, Tecnologia, Inovação e de desenvolvimento industrial do governo federal. Parágrafo } \\
\text { Único. A propriedade intelectual sobre os resultados obtidos pertencerá à empresa, na forma } \\
\text { da legislação vigente e de seus atos constitutivos. }\end{array}$ \\
\hline 3 & $\begin{array}{l}\text { Universidade Federal de } \\
\text { São João Del-Rei - } \\
\text { UFSJ }\end{array}$ & UFSJ & MG 2016 & $\begin{array}{l}\text { Resolução } \mathrm{n}^{\circ} 028 \text {, de } 19 \text { de } \\
\text { setembro de } 2016\end{array}$ & $\begin{array}{l}\text { CAPÍTULO V - DA TRANSFERÊNCIA DE TECNOLOGIA Art. 11. A UFSJ poderá } \\
\text { obter o direito de uso ou de exploração de criação protegida e participar minoritariamente de } \\
\text { capital social de empresa com o propósito de desenvolver inovação tecnológica, desde que } \\
\text { haja manifestação favorável, devidamente motivada, pelo Núcleo de Empreendedorismo e } \\
\text { Inovação Tecnológica, observadas as condições de limitações impostas pela legislação } \\
\text { vigente. }\end{array}$ \\
\hline 4 & $\begin{array}{c}\text { Universidade Federal de } \\
\text { Uberlândia }\end{array}$ & UFU & MG 2006 & $\begin{array}{c}\text { Resolução } \mathrm{n}^{\circ} 08 / 2006 \text {, do } \\
\text { Conselho Universitário }\end{array}$ & $\begin{array}{l}\text { VI - DO ESTÍMULO À CONSTRUÇÃO DE AMBIENTES ESPECIALIZADOS E } \\
\text { COOPERATIVOS DE INOVAÇÃO Art. } 11 \text { - A Universidade poderá participar } \\
\text { minoritariamente do capital de empresa de empresa privada de propósito específico, que vise } \\
\text { ao desenvolvimento de projetos científicos ou tecnológicos para a obtenção de produto ou } \\
\text { processo inovador, desde que haja previsão orçamentária e autorização do Presidente da } \\
\text { República. Parágrafo Único. A Propriedade Intelectual sobre os resultados obtidos pertencerá } \\
\text { às instituições detentoras do Capital Social, na proporção da respectiva participação. }\end{array}$ \\
\hline 5 & $\begin{array}{c}\text { Universidade Federal de } \\
\text { Viçosa }\end{array}$ & UFV & MG 2010 & $\begin{array}{l}\text { Resolução nº 06/2010 - } \\
\text { Conselho Universitário }\end{array}$ & $\begin{array}{l}\text { Da Participação da Universidade Federal de Viçosa no processo de Inovação Art. } 5^{\circ} \text { - A } \\
\text { UFV poderá participar minoritariamente do capital de empresa privada de propósito específico } \\
\text { que vise ao desenvolvimento de projetos científicos ou tecnológicos para obtenção de produtos } \\
\text { ou processos inovadores, desde que haja previsão orçamentária e autorização do Presidente } \\
\text { da República. }\end{array}$ \\
\hline 6 & $\begin{array}{l}\text { Instituto Federal de } \\
\text { Mato Grosso do Sul }\end{array}$ & IFMS & MS 2017 & $\begin{array}{c}\text { Resolução n }{ }^{\circ} \text { 054, de } 07 \\
\text { de julho de } 2017\end{array}$ & $\begin{array}{l}\text { CAPÍTULO VII - A PERMISSÃO DE USO E COMPARTILHAMENTO DE } \\
\text { INFRAESTRUTURA Art. } 16 \text { - O IFMS poderá, nos termos da legislação vigente, participar } \\
\text { minoritariamente do capital social de empresas, com o propósito de desenvolver produtos ou } \\
\text { processos inovadores que estejam de acordo com as diretrizes e prioridades definidas nas } \\
\text { políticas de ciência, tecnologia, inovação e de desenvolvimento industrial de cada esfera de } \\
\text { Governo. }\end{array}$ \\
\hline 7 & $\begin{array}{l}\text { Instituto Nac. } \\
\text { Metrologia, Qualidade e } \\
\text { Tecnologia }\end{array}$ & INMETRO & RJ 2017 & $\begin{array}{l}\text { REGULAMENTO DA } \\
\text { POLÍTICA DE } \\
\text { INOVAÇÃO DO } \\
\text { INMETRO } \\
\text { APROVADO PELA } \\
\text { PORTARIA PRESI } \\
\text { INMETRO n } 130 \text {, de } 17 \\
\text { de maio de } 2017\end{array}$ & $\begin{array}{l}13 \text { DISPOSIÇÕES GERAIS 13.4 A participação do Inmetro no capital social de empresas } \\
\text { deverá ser avaliada e deliberada pelo Comitê de Inovação e adotada por intermédio da } \\
\text { fundação de apoio, observada a legislação pertinente. }\end{array}$ \\
\hline 8 & $\begin{array}{c}\text { Universidade Federal de } \\
\text { Minas Gerais }\end{array}$ & UFMG & MG 2018 & $\begin{array}{c}\text { Portaria } \mathrm{n}^{\circ} 028, \text { de } 16 \text { de } \\
\text { março de } 2018\end{array}$ & $\begin{array}{l}\text { Reedita, com alterações, a Portaria } n^{\circ} 060 \text {, de } 22 \text { de agosto de 2011, que estabelece a } \\
\text { estrutura da Coodernadoria de Transferência de Tecnologia e Inovação (CTIT), } \\
\text { considerando a legislação vigente e a Política de Inovação da UFMG. Art. } 2^{\circ} \text { - A gestão e } \\
\text { implementação da política de inovação da UFMG é responsabilidade da CTIT, que ficará } \\
\text { vinculada diretamente ao Gabinete do Reitor. } \S 1^{\circ} \text { - A Diretoria da CTIT será exercida por } \\
\text { um Diretor e um Vice-diretor, ambos de livre indicação do Reitor. } \S 2^{\circ} \text { - A CTIT poderá ser } \\
\text { constituída com personalidade jurídica própria, em consonância com a legislação vigente. } \S 3^{\circ} \text {. } \\
\text { A UFMG fica autorizada a estabelecer parceria com a Fundação de Desenvolvimento da } \\
\text { Pesquisa (FUNDEP) para apoio à adequada implementação das competências e do } \\
\text { funcionamento da CTIT, por intermédio da celebração de termo jurídico específico para essa } \\
\text { finalidade. }\end{array}$ \\
\hline
\end{tabular}

Fonte: Desenvolvido pelos autores deste artigo, com dados extraídos das Resoluções das ICT

Como demonstrado no Quadro 2, de maneira geral, as ICT que possuem cláusulas de participação minoritária no capital social de empresas apresentam em suas resoluções o que a Lei n. 13.243, de 11 de janeiro de 2016, e o Decreto n. 9.283, de 7 de fevereiro de 2018, estabelecem na Seção II, Artigo $4^{\circ}$ do Decreto: 
Ficam as ICT públicas integrantes da administração pública indireta, as agências de fomento, as empresas públicas e as sociedades de economia mista autorizadas a participar minoritariamente do capital social de empresas, com o propósito de desenvolver produtos ou processos inovadores que estejam de acordo com as diretrizes e as prioridades definidas nas políticas de ciência, tecnologia, inovação e de desenvolvimento industrial.

As ICT apresentam no texto das resoluções a expressão "Poderá" e seguindo, "participar minoritariamente do capital social de empresa", e posteriormente, descrevem de maneira similar ao definido no texto da legislação.

A exceção é a UFMG, que não possui cláusulas de participação minoritária no capital social de empresas, mas aprovou a Portaria n. 028, de 16 de março de 2018, reeditando a Portaria n. 060, de 22 de agosto de 2011, que:

[...] estabelece a estrutura da Coodernadoria de Transferência de Tecnologia e Inovação (CTIT), considerando a legislação vigente e a Política de Inovação da UFMG.

Na reedição, a UFMG definiu no Artigo $2^{\circ}$, que:

A gestão e implementação da política de inovação da UFMG é responsabilidade da CTIT, que ficará vinculada diretamente ao Gabinete do Reitor. $\S 1^{\circ}$ - A Diretoria da CTIT será exercida por um Diretor e um Vice-diretor, ambos de livre indicação do Reitor. $\S 2^{\circ}$ - A CTIT poderá ser constituída com personalidade jurídica própria, em consonância com a legislação vigente. $\S 3^{\circ}$ - A UFMG fica autorizada a estabelecer parceria com a Fundação de Desenvolvimento da Pesquisa (FUNDEP) para apoio à adequada implementação das competências e do funcionamento da CTIT, por intermédio da celebração de termo jurídico específico para essa finalidade. (CTIT, 2018)

Com a aprovação da Portaria n. 028, a UFMG trouxe a possibilidade de parceria com a FUNDEP. A Fundação possui uma estrutura que apoia de forma direta iniciativas de empreendedorismo, iniciando na geração de ideias, programas de aceleração de startups e gestão de fundos de investimento:

a) Lemonade - Programa de pré-aceleração de startups, tem o intuito de desenvolver ideais e modelos de negócios (LEMONADE, 2018);

b) Techmall - A Fundep tem participação na Aceleradora de Negócios. A Techmall tem o foco no apoio, desenvolvimento e rápido crescimento de startups. A Techmall investe nas startups aceleradas e se torna sócia, até o desinvestimento, fase na qual a aceleradora vende sua participação para investidores ou para os sócios das startups (TECHMALL, 2018);

c) Fundepar - É uma gestora de Fundos de Investimento. A Fundepar lançou em 2018, o Fundo de Investimento em Participação "Seed4Science". O Fundo tem o objetivo de investir em empresas de base tecnológica inovadoras que estejam em estágio inicial de desenvolvimento (FUNDEPAR, 2018). 
Assim, com a possibilidade de parceria com a Fundação, a UFMG atuará de forma ativa na gestão da inovação e do emprendedorismo, incentivando a criação e desenvolvimento de startups e empresas de base tecnológica, com a opção de participação minoritária no capital social.

\section{Considerações Finais}

A partir do levantamento e análise da revisão bibliográfica, foi constatado que, apesar dos avanços que a Lei n. 10.973/2004 - Lei de Inovação, e mais recentemente, a Lei n. 13.243, o novo marco legal de Ciência, Tecnologia e Inovação, e todos os esforços de investimentos em ciência, tecnologia e inovação, o Brasil ainda não conseguiu transbordar as pesquisas desenvolvidas nas ICT para o mercado.

As informações reafirmam as impressões sobre os resultados dos investimentos e iniciativas de interação universidade-empresa-governo realizadas no sistema nacional de inovação brasileiro na última década, e, de acordo com Stal e Fujino (2005), o modelo de tríplice hélice no Brasil ainda é embrionário. As ICT têm vários papéis, dentre eles a formação de recursos humanos qualificados, a extensão e a pesquisa, mas é essencial a ampliação dos resultados da pesquisa, transferência de tecnologia para as empresas e a criação de startups.

Os resultados de participação minoritária no capital social das ICT em empresas é um tema recente (introduzido na Lei n. 13.243/2016 e regulamentado no Decreto n. 9.283/2018), tanto que apenas sete ICT possuem cláusulas de participação minoritária no capital social. E as instituições que possuem cláusulas, não apresentam a forma de atuação regulamentada nos Art. $4^{\circ}$, $\S 1^{\circ}$ do Decreto. As ICT apresentam em suas resoluções internas apenas uma repetição do que a legislação define e não atuam de forma direta para seleção e investimento em empresas de base tecnológica. Como exceção foi identificada a UFMG, que abriu a possibilidade de parceria com a FUNDEP para o fortalecimento e implantação da política de inovação da instituição.

Assim, é necessária uma maior articulação entre os entes do Sistema Nacional de Inovação para o desenvolvimento e implantação de iniciativas de investimentos ligados a ICT e, essencialmente, que as mesmas tenham segurança jurídica e institucional para participação acionária em empresas de base tecnológica.

As associações como Fortec, Anprotec e ABVCAP precisam se articular para oferecer treinamentos, workshop e oficinas, com o objetivo de capacitar gestores para a atuação no tema e produzir material informativo, como: cartilhas e guias para que as ICT tenham informações sobre como implantar ações de investimentos em empresas que nascem nos seus ambientes de inovação.

\section{Referências}

ABDI - AGÊNCIA BRASILEIRA DE DESENVOLVIMENTO INDUSTRIAL. Introdução ao private equity e venture capital para empreendedores. Brasília, 2011. 310 p.

ANPEI - ASSOCIAÇÃO NACIONAL DE PESQUISA E DESENVOLVIMENTO DAS EMPRESAS INOVADORAS. Mapa do Sistema Brasileiro de Inovação. Disponível em: http://anpei.org.br/ publicacoes/mapas-da-inovacao/. Acesso em: 3 jul. 2018. 
ANPROTEC - ASSOCIAÇÃO NACIONAL DE ENTIDADES PROMOTORAS DE EMPREENDIMENTOS INOVADORES. Lista de associados. Disponível em: http://anprotec.org.br/ site/menu/a-anprotec/associados-anprotec/. Acesso em: 8 fev. 2018.

AUTM. Highlightsof AUTM's U.S. Licensing Activity Survey FY2015, 2015. 12 p.

BM\&F-BOVESPA - Bolsa de Mercadorias e Futuro - Bolsa de Valores de São Paulo. Fundos de Investimentos em Participação. Disponível em: http://www.bmfbovespa.com.br/pt_br/produtos/ listados-a-vista-e-derivativos/renda-variavel/fundos-de-investimento-em-participacoes-fip.htm. Acesso em: 10 jun. 2018.

BRASIL. Lei n. 13.243, de 11 de janeiro de 2016. Dispõe sobre estímulos ao desenvolvimento científico, à pesquisa, à capacitação científica e tecnológica e à inovação e altera a Lei n. 10.973, de 2 de dezembro de 2004, a Lei n. 6.815, de 19 de agosto de 1980, a Lei n. 8.666, de 21 de junho de 1993, a Lei n. 12.462, de 4 de agosto de 2011, a Lei n. 8.745, de 9 de dezembro de 1993, a Lei n. 8.958, de 20 de dezembro de 1994, a Lei n. 8.010, de 29 de março de 1990, a Lei n. 8.032, de 12 de abril de 1990, e a Lei n. 12.772, de 28 de dezembro de 2012, nos termos da Emenda Constitucional no 85, de 26 de fevereiro de 2015. Brasília: Diário Oficial da União, 2016.

BRASIL. Decreto n. 9.283, de 7 de fevereiro de 2018. Regulamenta a Lei n. 10.973, de 2 de dezembro de 2004, a Lei n. 13.243, de 11 de janeiro de 2016, o art. 24 , $\S 3^{\circ}$, e o art. 32 , $\S 7^{\circ}$, da Lei n. 8.666, de 21 de junho de 1993, o art. $1^{\circ}$ da Lei n. 8.010, de 29 de março de 1990, e o art. $2^{\circ}$, caput, inciso I, alínea "g", da Lei n. 8.032, de 12 de abril de 1990, e altera o Decreto n. 6.759, de 5 de fevereiro de 2009, para estabelecer medidas de incentivo à inovação e à pesquisa científica e tecnológica no ambiente produtivo, com vistas à capacitação tecnológica, ao alcance da autonomia tecnológica e ao desenvolvimento do sistema produtivo nacional e regional. Brasília, 2018.

CTIT - Coodernadoria de Transferência de Tecnologia e Inovação. Portaria n. 028, de 16 de março de 2018. Reedita, com alterações, a Portaria n. 060, de 22 de agosto de 2011, que estabelece a estrutura da Coodernadoria de Transferência de Tecnologia e Inovação (CTIT), considerando a legislação vigente e a Política de Inovação da UFMG. Disponível em: http://www.ctit. ufmg.br/publicacoes-e-editais/. Acesso em: 9 jul. 2018.

ETZKOWITZ, H. The norm of entrepreneurial science: cognitive effects of the new Universityindustry linkages. Research Policy, [S.I.], v. 27, n. 8, p. 823-833, 1998.

ETZKOWITZ, H. The evolution of the entrepreneurial university. Int. Journal Technological and Globalization, [S.l.], v. 1, n. 1, p. 64 - 77, 2004.

ETZKOWITZ, H.; LEYDESDORFF, L. The dynamics of innovation: from National Systems and "Mode 2" to the triple helix of University-Industry- Government relations. Research Policy, [S.I.], v. 29, n. 2, p. 109-123, 2000. Disponível em: https://www.sciencedirect.com/ search?pub $=$ Research\%20Policy\&volume $=29 \&$ page $=109 \&$ show $=25 \&$ sortBy $=$ relevance $\&$ origin $=$ jr nl_home\&zone $=$ search\&cid=271666. Acesso em: 12 jun. 2018.

ETZKOWITZ, H.; KLOFSTEN, M. The innovation region: toward a theory of knowledge - based regional development. R\&D Management, [S.l.], v. 35, n. 3, p. 243 -255, 2005. Disponível em: https://onlinelibrary.wiley.com/doi/pdf/10.1111/j.1467-9310.2005.00387.x. Acesso em: 5 jun. 2018.

FINEP - FINANCIADORA DE ESTUDOS E PROJETOS. Glossário. Disponível em: http://www. finep.gov.br/biblioteca/glossario. Acesso em: 23 jan. 2018. 
FREEMAN, C. The National Systems of Innovation in historical perspective. Cambridge Journal of Economics, [S.1.], v. 19, n. 1, p. 5-24, Feb. 1995. Disponível em: https://academic.oup.com/cje/ article-abstract/19/1/5/1708372?redirectedFrom=PDF. Acesso em: 4 jul. 2018.

FUNDEPAR. Fundo de Investimentos em Participações - Seed4Science. Disponível em: http://fundepar.com.br/seed4science/. Acesso em: 9 jul. 2018.

GIL, A. C. Como elaborar projetos de pesquisa. 4. ed. São Paulo: Atlas, 2007. 176 p.

PLONSKI, G. A. Cooperação empresa-universidade: antigos dilemas, novos desafios. Revista USP, São Paulo, v. 25, p. 32- 41, mar./maio, 1995.

LEMONADE. Programa de Pré-aceleração de Startups. Disponível em: http:// programalemonade.com/o-programa/\#o-que-e-o-lemonade. Acesso em: 9 jul. 2018.

MARGINSON, S.; CONSIDINE, M. The enterprise university - power, governance and reinvention in Australia. Cambridge: Cambridge University Press, 2004. 272p.

MATTOS, P. T. L.; ABDAL, A. Inovação: estratégia de sete países. Brasília, ABDI, 2010. 343 p. (Série Cadernos da Indústria ABDI, v. XV)

MCTIC - MINISTÉRIO DA CIÊNCIA, TECNOLOGIA, INOVAÇÃO E COMUNICAÇÃO.

Indicadores CT\&I. Disponível em: http://www.mctic.gov.br/mctic/opencms/indicadores/ indicadores_cti.html. Acesso em: 25 jan. 2018.

MCTIC - MINISTÉRIO DA CIÊNCIA, TECNOLOGIA, INOVAÇÃO E COMUNICAÇÃO. Formulário para Informações sobre a Política de Propriedade Intelectual das Instituições Científicas, Tecnológicas do Brasil (FORMICT) ano-base 2016. Brasília, 2017. Disponível em: http://www.mctic.gov.br/mctic/export/sites/institucional/tecnologia/propriedade_intelectual/ arquivos/Relatorio-Formict-Ano-Base-2016.pdf. Acesso em: 26 jun. 2018.

MEIRELLES, J. L. F.; PIMENTA JR, T; DO NASCIMENTO REBELATTO, D. A. Venture capital e private equity no Brasil: Alternativa de financiamento para empresas de base tecnológica [Venture capital and private equity in Brazil: Alternative of

financing for high technology industries. Gestão e Produção, [S.l.], v. 15, n. 1, p. 11-21. jan. 2008.

PLONSKI, G. A. Cooperação empresa-universidade: antigos dilemas, novos desafios. Revista USP, São Paulo, v. 25, p. 32- 41, mar./maio, 1995.

RAUEN, C. V. O Novo Marco Legal da Inovação no Brasil: O que muda na relação ICTEmpresa? Radar: Tecnologia, Produção e Comércio Exterior. Brasília: IPEA - Instituto de Pesquisa Econômica Aplicada, n. 43, p. 21-35, 2016.

SÁBATO, J. A.; BOTANA, N. La ciencia y la tecnología en el desarrollo futuro de América Latina. Revista de la Integración, Buenos Aires, ano 1, n. 3, 1968. Disponível em: http://docs.politicascti. net/documents/Teoricos/Sabato_Botana.pdf. Acesso em: 26 jun. 2018.

STAL, E.; FUJINO A. As relações Universidade-Empresa no Brasil sob a ótica da Lei de Inovação. RAI - Revista de Administração e Inovação, São Paulo, v. 2, n. 1, p. 5-19, 2005.

TECHMALL. Programa de Aceleração de Startups. Disponível em: http://techmallsa.com.br/ aceleracao/. Acesso em: 9 jul. 2018. 


\section{Sobre os Autores}

\section{Leonardo Miranda Frossard}

E-mail: leonardo.frossard@ufjf.edu.br Mestrado

Endereço profissional: Rua José Lourenço Kelmer, S/N, Bairro Martelos, Campus Universitário, Prédio do Critt, Juiz de Fora, MG. CEP: 36036-900.

\section{Rita Pinheiro-Machado}

E-mail: ritap@inpi.gov

Doutorado

Endereço profissional: Rua Mayrink Veiga, 9/17 andar, Centro, Rio de Janeiro, RJ. CEP: 20090-910.

\section{Flávia Lima do Carmo}

E-mail: flaviacarmo@inovacao.ufrj.br Pós-Doutorado

Endereço profissional: Rua Hélio de Almeida, s/n, Incubadora de Empresas da Coppe, prédio 2, sala 29, Cidade Universitária, Ilha do Fundão, Rio de Janeiro, RJ. CEP: 21941-614. 


\section{Anexo A - Quadro de ICTs respondentes do FORMICT e associadas à ANPROTEC}

\begin{tabular}{|c|c|c|c|c|c|c|c|c|}
\hline ICT & SIGLA & UF & Site do NIT & $\begin{array}{l}\text { Associada a } \\
\text { Anprotec? }\end{array}$ & $\begin{array}{c}\text { Resolução } \\
\text { Encontrada? }\end{array}$ & $\begin{array}{c}\text { Possui } \\
\text { Politica de } \\
\text { Inovação? }\end{array}$ & \begin{tabular}{|c|} 
Possui \\
Politica de \\
Pl?
\end{tabular} & $\begin{array}{l}\text { Possui } \\
\text { Cláusulas de } \\
\text { Participação } \\
\text { Acionária? }\end{array}$ \\
\hline Universidade Estadual de Ciências da Saúde de Alagoas & UNCISAL & $\mathrm{AL}$ & https://nit.uncisal.edu.br// & Sim & Sim & \begin{tabular}{|l|l|l} 
Não \\
\end{tabular} & Sim & Não \\
\hline Universidade Federal de Alagoas & UFAL & AL & http://www.ufal.edu.br/pesquisa-e-inovacao/ino & Sim & Sim & Não & Sim & Não \\
\hline Instituto de Desenvolvimento Sustentável Mamirauá & IDSM & AM & https://www.mamiraua.org.br/pt-br/institucional & Sim & Não & Não & Não & Não \\
\hline Instituto Federal do Amazonas & IFAM & AM & http://www2.ifam.edu.br/pro-reitorias/pesquisa- & Sim & Sim & Não & Sim & Não \\
\hline Centro de Pesq. e Desen. Tec. em Infor. de llhéus & CEPEDI & BA & http://www.cepedi.org.br & Sim & Não & Não & Não & Não \\
\hline Universidade Federal da Bahia & UFBA & $B A$ & https://propci.ufba.br/npi & sim & Sim & Não & Sim & Não \\
\hline Universidade Federal do Ceará & UFC & CE & http://www.cit.ufc.br/pt/legislacao/ & sim & Sim & sim & Não & sim \\
\hline Instituto Federal do Espirito Santo & IFES & ES & http://incubadora.sr.ifes.edu.br/http://prppg.ifes & Sim & Não & & - & - \\
\hline Universidade Federal do Espirito Santo & UFES & ES & http://www.prppg.ufes.br/normas-e-resolucōes & sim & Sim & Não & sim & Não \\
\hline Instituto Federal de Goiás & IFG & GO & https://www.ifg.edu.br/cite?showall=\&limitstart= & $=\operatorname{sim}$ & $\operatorname{sim}$ & Não & Sim & Não \\
\hline Centro Federal de Educação Tecnológica de MG & CEFET-MG & MG & http://www.cit.cefetmg.br// & sim & Sim & sim & Não & $\operatorname{sim}$ \\
\hline Centro Universitário de Patos de Minas & UNIPAM & MG & https://unipam.edu.br/site/nucleos.php & sim & Não & - & - & - \\
\hline Fundação Instituto Nacional de Telecomunicaçōes & Inatel & MG & http://inatel.br/empreendedorismo/quero-empre & Sim & Não & & - & - \\
\hline Universidade do Estado de Minas Gerais & UEMG & MG & http://nit.uemg.br/?page_id=258 & Sim & Não & Não & sim & Não \\
\hline Universidade Estadual de Montes Claros & UNIMONTES & MG & http://www.agora.unimontes.br/index.php & sim & Não & Não & Não & Não \\
\hline Universidade Federal de Itajubá & UNIFEI & MG & https://unifei.edu.br/nucleo-inovacao-tecnologic & Sim & Sim & Não & Sim & Não \\
\hline Universidade Federal de Juiz de Fora & UFJF & MG & www.uff.,br/critt & sim & Sim & Não & sim & Não \\
\hline Universidade Federal de Lavas & UFLA & MG & http://www.nintec.ufla.br/ & Sim & Não & Não & Sim & Não \\
\hline Universidade Federal de Minas Gerais & UFMG & MG & http://www.ctit.ufmg.br/ & Sim & Sim & sim & Não & Não \\
\hline Universidade Federal de Ouro Preto & UFOP & MG & http://nite.ufop.br/ & Sim & Não & - & - & - \\
\hline Universidade Federal de São João Del-Rei - UFSJ & UFSJ & MG & https://ufsi.edu.br/copin/regimento.php & Sim & Sim & sim & Não & Sim \\
\hline Universidade Federal de Uberlândia & UFU & MG & http://www.intelecto.ufu.br/legislacao.htm & sim & Sim & Não & Sim & sim \\
\hline \begin{tabular}{|l|} 
Universidade Federal de Viçosa \\
\end{tabular} & UFV & MG & http://www.cppi.ufv.br/pt-BR/politica-de-pi-na-u & Sim & Sim & Não & Sim & Sim \\
\hline Fundação Universidade Federal de Mato Grosso do Sul & UFMS & MS & https://aginova.ufms.br/nit/ & Sim & Não & Não & Não & Não \\
\hline Instituto Federal de Mato Grosso do Sul & IFMS & MS & http://www.ifms.edu.br/assuntos/pesquisa/emp. & Sim & Sim & sim & Não & Sim \\
\hline Instituto Federal de Mato Grosso & IFMT & MT & http://proex.ifmt.edu.br/conteudo/pagina/ativa-i & $\operatorname{sim}$ & Sim & Sim & Não & Não \\
\hline Universidade Federal de Mato Grosso & UFMT & MT & http://www.ufmt.br/ufmt/un/secao/11566/eit & Sim & Sim & sim & Não & Não \\
\hline Universidade Federal de Campina Grande - UFCG & NITT/UFCG & $\mathrm{PB}$ & http://www.nitt.ufcg.edu.br/sobre-o-nitt-ufcg// & sim & Sim & Näo & Sim & Não \\
\hline Associação Instituto de Tecnologia de Pernambuco & ITEP/OS & $\mathrm{PE}$ & http://www.itep.br/nucleo-de-inovacao-tecnolog & $\operatorname{sim}$ & Não & - & - & - \\
\hline Instituto Federal de Educação, Ciência e Tecnologia & IF SERTÃO-PE & $\mathrm{PE}$ & http://nit.ifsertao-pe.edu.br/ & $\operatorname{sim}$ & Não & & - & - \\
\hline Universidade Federal de Pernambuco & UFPE & PE & https://www.ufpe.br/positiva & sim & Sim & Sim & Não & Não \\
\hline Universidade Federal Rural de Pernambuco & UFRPE & $P E$ & http://www.nit.ufrpe.br/ & Sim & Não & - & - & - \\
\hline Instituto Federal do Piaui & IFPI & $\mathrm{PI}$ & https://www5.ifpi.edu.br/nit/index.php/apresent & Sim & Sim & Não & Sim & Não \\
\hline Fundação Parque Tecnologico Itaipu - Brasil & FPTI-BR & PR & https://www.pti.org.br/pt-br & sim & Não & - & - & - \\
\hline Instituto de Tecnologia do Paraná & TECPAR & PR & http://nitpar.pr.gov.br// & Sim & Não & - & - & - \\
\hline Pontificia Universidade Católica do Paraná & PUCPR & $P R$ & http://nitpar.pr.gov.br/ & Sim & Não & - & - & - \\
\hline Universidade Tecnológica Federal do Paraná & UTFPR & $P R$ & http://www.utfpr.edu.br/estrutura-universitaria/ & Sim & Sim & Não & Sim & Não \\
\hline Centro Fed. de Educ. Tec. Celso Suckow da Fonseca & CEFET/RJ & RJ & http://dippg.cefet-rj.br/index.php?option=com_c & Sim & Sim & $\operatorname{sim}$ & Não & Não \\
\hline Faculdades Católicas / PUC-Rio & PUC-Rio & RJ & http://www.agi.puc-rio.br/web/ & sim & Não & & & \\
\hline Instituto Nac. Metrologia, Qualidade e Tecnologia & INMETRO & RJ & http://www.inmetro.gov.br/inovacao/servico-ppi & Sim & Sim & Sim & Não & Sim \\
\hline Universidade do Estado do Rio de Janeiro & UERJ & RJ & http://www.inovueri.sr2.ueri.br/portal// & Sim & Não & & - & - \\
\hline Universidade Estadual do Norte Fluminense Darcy Ribeiro & UENF & RJ & http://uenf.br/reitoria/agenciainovacao/ & Sim & Sim & Não & Sim & Não \\
\hline Universidade Federal do Rio de Janeiro & UFRJ & RJ & http://www.inovacao.ufri.br/index.php/sobre-ag & Sim & Sim & Não & Sim & Não \\
\hline Universidade Federal Fluminense & UFF & RJ & http://www.etco.uff.br/ & Sim & Sim & Não & Sim & Não \\
\hline Universidade Federal Rural do Rio de Janeiro & UFRRJ & RJ & http://institucional.ufrri.br/nit// & Sim & Sim & Sim & Não & Não \\
\hline Instituto Federal de Educação, Ciência e Tecnologia & IFRN & $\mathrm{RN}$ & http://portal.ifrn.edu.br/pesquisa/nit & Sim & $\operatorname{sim}$ & Não & Sim & Não \\
\hline Universidade do Estado do Rio Grande do Norte & UERN & RN & http://di.uern.br/nit/ & Sim & Não & & & \\
\hline Universidade Federal do Rio Grande do Norte & UFRN & $\mathrm{RN}$ & http://www.nit.ufrn.br/ & Sim & Sim & Não & Sim & Não \\
\hline Universidade Federal Rural do Semi-Árido & UFERSA & $\mathrm{RN}$ & https://nit.ufersa.edu.br/ & sim & Sim & Não & Sim & Não \\
\hline Instituto Federal de Educação, Ciência e Tecnologia de Rondônia & IFRO & $\mathrm{RO}$ & http://portal.ifro.edu.br/organograma/59-conteug & SSim & Não & - & - & - \\
\hline Fund. Vale do Taquari de Educ. e Desenv. Social & FUVATES & RS & https://www.univates.br/pesquisa & sim & Não & - & - & - \\
\hline Fundação de Ciência e Tecnologia & CIENTEC & RS & http://www.cientec.rs.gov.br/?model=conteudo\& & Sim & Não & - & - & - \\
\hline Fundação Universidade de Caxias do Sul & UCS & RS & https://www.ucs.br/site/pesquisa-inovacao-e-des & Sim & Sim & Não & Sim & Não \\
\hline Fundação Universidade Federal de Pelotas & UFPel & RS & https://wp.ufpel.edu.br/cit/ & sim & Não & & - & - \\
\hline Pontificia Universidade Católica do RS & PUCRS & RS & http://www.pucrs.br/tecnopuc/ & Sim & Sim & Não & Sim & Não \\
\hline Universidade de Passo Fundo & UPF & RS & http://upftec.upf.br/ & Sim & Não & - & - & - \\
\hline Universidade de Santa Cruz do Sul & UNISC & RS & https://www.unisc.br/pt/home/estrutura-adminis & Sim & Sim & Não & Sim & Não \\
\hline Universidade do Vale do Rio dos Sinos & UNISINOS & RS & http://www.unisinos.br/pesquisa-e-inovacao/nitt & $\operatorname{sim}$ & Sim & Não & Sim & Não \\
\hline Universidade Federal de Santa Maria & UFSM & RS & http://w3.ufsm.br/agittec/ & Sim & Sim & Sim & Não & Não \\
\hline Universidade Federal do Rio Grande & FURG & RS & http://www.propesp.furg.br/ & Sim & Sim & Näo & Sim & Não \\
\hline Universidade Federal do Rio Grande do Sul & UFRGS & RS & http://nitec.co/pt/ & Sim & Não & & & \\
\hline Universidade Feevale & Feevale & RS & https://www.feevale.br/institucional/bem-vindo & sim & $\operatorname{sim}$ & Não & $\operatorname{sim}$ & Não \\
\hline Universidade Luterana do Brasil & ULBRA & RS & http://ulbratech.com.br/v1/nitts/ & sim & Não & & - & \\
\hline Universidade Regional do Noroeste do Estado do RS & UNIJUÍ & RS & http://www.unijui.edu.br/pesquisa/inovacao-tect & Sim & Não & - & - & - \\
\hline Universidade Regional Integrada & URI-Frederico Westphalen & RS & http://www.fw.uri.br/site/pagina/nucleo-de-ino & Sim & Não & - & - & - \\
\hline Fundação CERTI & CERTI & $\mathrm{sc}$ & http://www.certi.org.br/pt/servicoseprodutos-prg & Sim & Não & - & - & - \\
\hline Fundação Educacional da Região de Joinville & UNIVILLE & sc & http://www.univille.edu.br/pt br/institucional/p & Sim & Sim & Sim & Não & Não \\
\hline Fundação Universidade do Vale do Itajai & UNIVALI & $\mathrm{sc}$ & https://www.univali.br/institucional/vice-reitoria & Sim & Não & & & \\
\hline Universidade Comunitária da Região de Chapecó & Unochapecó & sc & https://www.unochapeco.edu.br/nitt & Sim & Sim & Não & Sim & Não \\
\hline Universidade do Extremo Sul Catarinense & UNESC & sc & http://www.unesc.net/portal/capa/index/260 & sim & $\operatorname{sim}$ & Sim & Não & Não \\
\hline Universidade do Sul de Santa Catarina & Unisul & sc & http://www.unisul.br/wps/portal/home/pesquise & Sim & Não & & - & \\
\hline Universidade Federal de Santa Catarina & UFSC & $\mathrm{sc}$ & http://sinova.ufsc.br/legislacao/legislacao-ufsc/ & Sim & Sim & Não & Sim & Não \\
\hline Centro de Tecnologia da Informação Renato Archer & CTI Renato Archer & SP & http://www.nitmantiqueira.org.br/portal/index.p & Sim & Não & - & - & - \\
\hline FACENS- Faculdade de Engenharia de Sorocaba: IPEAS & IPEAS & SP & http://www.facens.br/ & Sim & Não & - & - & - \\
\hline Fundação Instituto Pólo Avançado da Saúde de RP & FIPASE & SP & http://superaparque.com.br/nit/ & Sim & Não & & - & - \\
\hline Fundação Universidade Federal de São Carlos & UFSCar & SP & http://www.inovacao.ufscar.br/ & $\operatorname{sim}$ & Sim & Não & $\operatorname{sim}$ & Não \\
\hline Fundação Universidade Federal do ABC - UFABC & UFABC & SP & http://nti.ufabc.edu.br/ & Sim & Não & & - & \\
\hline Fundação Valeparaibana de Ensino & FVE/UNIVAP & $\mathrm{SP}$ & http://www.nitmantiqueira.org.br/portal/index.p & Sim & Não & - & - & - \\
\hline Instituto de Pesquisas Tecnológicas do Estado de São Paulo & IPT & SP & http://www.ipt.br/patentes & sim & Não & & - & - \\
\hline Universidade de São Paulo & USP & SP & http://www.inovacao.usp.br// & Sim & Não & - & - & - \\
\hline Universidade Estadual de Campinas & UNICAMP & SP & https://www.inova.unicamp.br/legislacao/ & sim & Sim & Não & Sim & Não \\
\hline
\end{tabular}

Fonte: Desenvolvido pelos autores, com dados do MCTIC e Anprotec 